$05.1 ; 06.5$

\title{
Необычный механизм релаксации напряжений несоответствия В тонких нанопленках
}

\author{
(C) Е.М. Труханов, С.А. Тийс \\ Институт фозики полупроводников им. А.В. Ржанова СО РАН, Новосибирск, Россия \\ E-mail: trukh@isp.nsc.ru
}

Поступило в Редакцию 24 мая 2019 г.

В окончательной редакции 15 июля 2019г.

Принято к публикации 5 августа 2019г.

\begin{abstract}
Установлен новый механизм релаксации напряжений несоответствия в нанопленках при изменении плотности поверхностных фаз. Он обеспечивается упорядоченным массопереносом атомов из напряженного атомного слоя. В процессе фазового перехода $7 \times 7 \rightarrow 5 \times 5$ в пленке $\mathrm{Ge}$ на $\mathrm{Si}(111)$ происходит частичная компенсация сжимающих напряжений на границе объемного кристалла и сверхструктуры за счет растягивающей деформации в неплотных слоях поверхностной фазы.
\end{abstract}

Ключевые слова: германий, механизм релаксации, сверхструктурный переход, массоперенос.

DOI: 10.21883/PJTF.2019.22.48645.17893

Для решения научных и технологических задач в полупроводниковой наноэлектронике получили распространение эпитаксиальные гетеросистемы с границами раздела (111) [1]. В этой работе структурные исследования наиболее детально выполнены для наносистемы $\mathrm{GeSi}(111)$. Установлено, что на начальной стадии формирования пленки $\mathrm{Ge}$ возникают двумерные островки высотой не более $1 \mathrm{~nm}$. В квазиравновесных условиях роста островки разрастаются в латеральных направлениях, сохраняя постоянную высоту [2]. Для подавляющего большинства островков $\mathrm{Ge}$ сначала на их поверхности повторяется сверхструктурная фаза чистой поверхности кремния $7 \times 7$. По мере латерального разрастания до размеров $(\sim 20-30 \mathrm{~nm})$, в десятки раз превышающих размер элементарной ячейки $7 \times 7$, на поверхности островков начинает формироваться структура $5 \times 5$. К моменту коалесценции островков в сплошной смачивающий слой $\mathrm{Ge}$ структура $5 \times 5$ занимает уже $100 \%$ поверхности [2]. Поскольку толщина пленки является докритической для введения дислокаций несоответствия, в таких условиях невыгодно протекание пластической релаксации напряжений, и в литературе обычно рассматривается уменьшение только упругой энергии гетеросистемы. В данном исследовании установлен новый механизм релаксаций напряжений, обусловленный протеканием перехода поверхностных фаз.

На рис. 1 приведены изображения сканирующего туннельного микроскопа (СТМ) поверхностных фаз $7 \times 7$ и $5 \times 5$, совмещенные с атомными моделями соответствующих элементарных ячеек $7 \times 7$ и $5 \times 5$. На всех СТМ-картинах темные области соответствуют углублениям, а светлые - выступающим элементам рельефа. На рис. $1, a, b$ для атомных схем фаз $7 \times 7$ и $5 \times 5$ представлен вид сверху, а на рис. 1, $c$ - их поперечные сечения. Три верхних неполных атомных слоя зарождающихся островков Ge занимает поверхностная фаза (ПФ; англ. вариант SP - surface phase), а четыре нижних слоя представляют собой полноценный кристалл $\mathrm{Ge}$ в виде напряженной псевдоморфной прослойки (ПП; англ. вариант PI - pseudomorphic interlayer) между подложкой кремния и ПФ пленки.

Обсудим структуру упомянутых поверхностных фаз. В верхнем (первом) слое присутствуют только дополнительные атомы Ge (адатомы). Положение адатомов не соответствует позициям алмазной решетки, поэтому они изображены на рис. 1 наиболее крупными сферами и указаны штриховыми стрелками. Их число равно 12 и 6 для ячеек $7 \times 7$ и $5 \times 5$ соответственно. Поскольку адатомы выступают над поверхностью, на СТМ-изображениях они видны в виде ярких светлых пятен. В среднем (втором) слое указанных ячеек находится 42 и 20 атомов, а в нижнем (третьем) слое - 48 и 24 атома соответственно. Таким образом, атомные структуры поверхностных фаз представляют собой три незаполненных атомных слоя (слои $1-3$ на рис. $1, c)$, которые в сумме содержат 102 и 50 атомов для ячеек $7 \times 7$ и $5 \times 5$ соответственно. Особенностью обоих вариантов поверхностных фаз являются глубокие угловые вакансии (corner holes), расположенные в вершинах ромбов (рис. $1, a, b)$ и пронизывающие все три слоя сверхструктуры (указаны сплошными стрелками на рис. $1, c)$. На СТМ-изображениях это самые крупные темные участки. На рис. 2 они схематично изображены в виде узких вертикальных светлых полосок. Необходимо отметить, что из-за этих глубоких вакансий нижние (третьи) слои поверхностных фаз содержат не 49 или 25 атомов, а на один атом меньше по сравнению со слоями в псевдоморфной прослойке или по сравнению с объемом подложки. Как показывает расчет, атомные плотности нижних слоев ПФ, приходящиеся на единицу площади границы раздела между ПФ и ПП, составляют $\rho_{7 \times 7}=7.67$ atom $/ \mathrm{nm}^{2}$ и $\rho_{5 \times 5}=7.52$ atom $/ \mathrm{nm}^{2}$, что мень- 


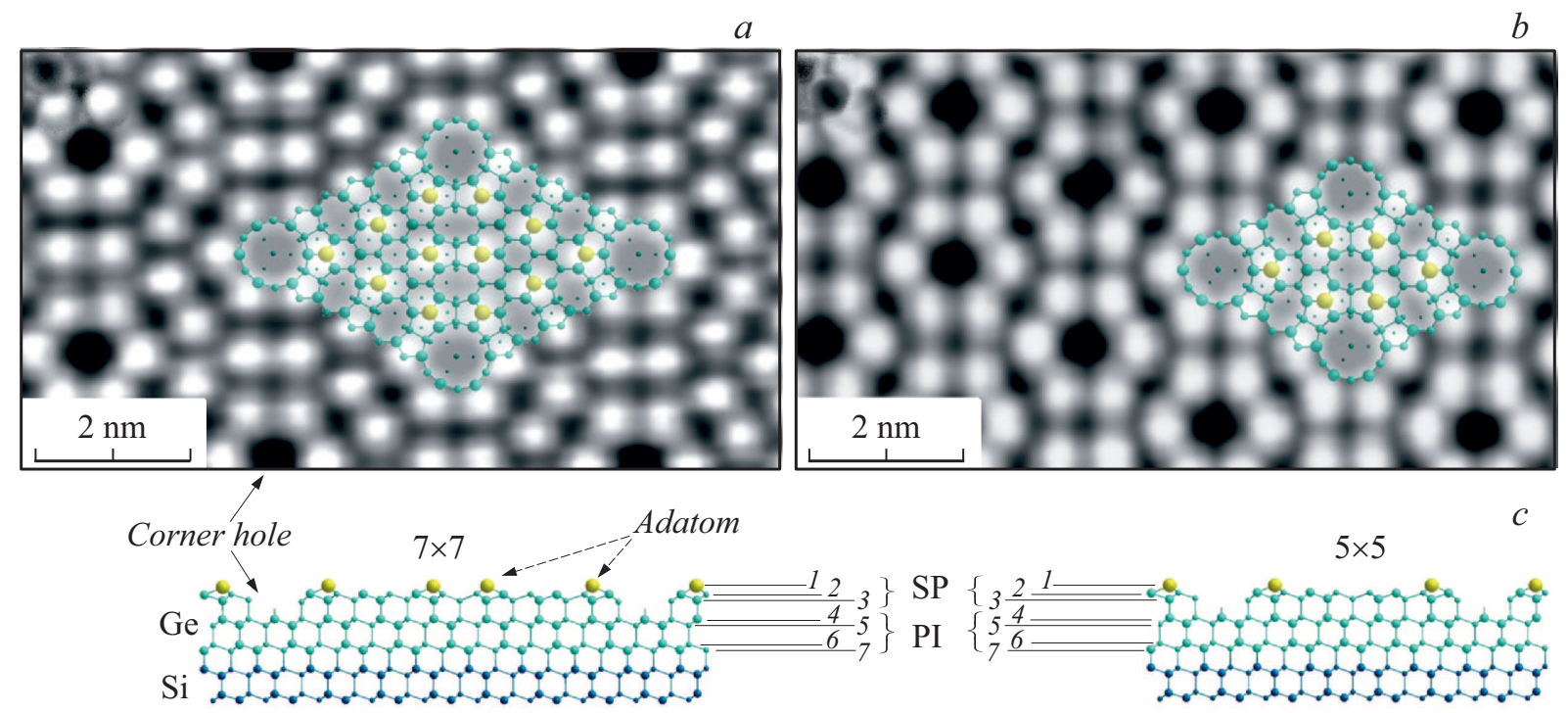

Рис. 1. СТМ-изображения поверхностных фаз $7 \times 7(a)$ и $5 \times 5(b)$ со схемами элементарных ячеек, а также соответствующие схемы поперечных срезов ячеек вдоль длинной диагонали ромба (c). Цифрами 1-3 обозначены номера атомных слоев поверхностной фазы (SP), цифрами 4-7 - псевдоморфной прослойки (PI). Штриховыми стрелками показаны адатомы, сплошными стрелками - угловые вакансии.

ше объемной плотности $\rho_{1 \times 1}=7.83$ atom $/ \mathrm{nm}^{2}$. Поскольку поверхностная фаза $5 \times 5$ является более „рыхлой“, происходит фазовый переход $7 \times 7 \rightarrow 5 \times 5$ (рис. 2), который способствует снижению сжимающих напряжений пленки $\mathrm{Ge}$ на $\mathrm{Si}(111)$. При фазовом переходе формируется новая система более плотно упорядоченных глубинных вакансий структуры $5 \times 5$ и происходит дополнительная релаксация напряжений. Необходимо отметить, что увеличение плотности вакансий сопровождается высвобождением избыточных атомов $\mathrm{Ge}$, которые за счет массопереноса перемещаются и встраиваются в

\section{$7 \times 7$}
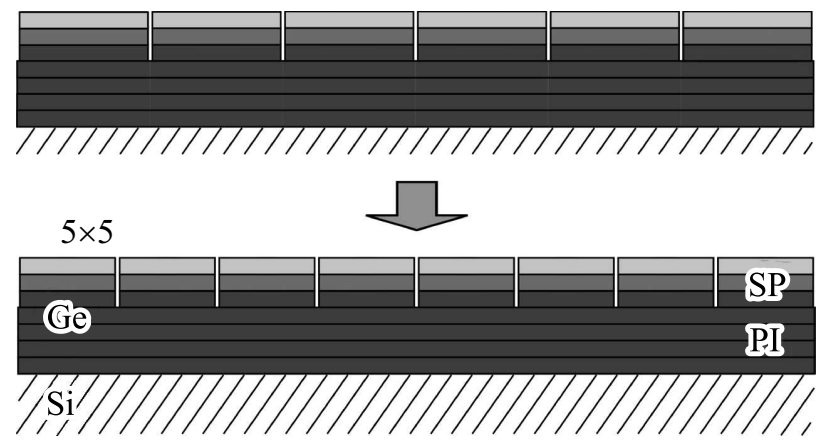

Рис. 2. Схематическое изображение фазового перехода $7 \times 7 \rightarrow 5 \times 5$ пленки Ge на подложке $\mathrm{Si}$. Поперечные сечения выполнены вдоль длинной диагонали ромба соответствующих элементарных ячеек (рис. 1). Три верхних слоя принадлежат поверхностной фазе (SP), а четыре нижних полных слоя псевдоморфной прослойке (PI). Степень черноты закрашенных площадей атомных слоев SP характеризует их атомную плотность. края островков, ускоряя их латеральный рост. Такой вид релаксации напряжений связан с дальнодействующим поверхностным массопереносом в отличие от процесса введения дислокаций.

Представленные результаты исследований показывают, что в исследуемом случае процесс фазового перехода является механизмом релаксации напряжений несоответствия. Переход начинается, когда в результате латерального разрастания островка его площадь приблизительно на два порядка величины превышает площадь элементарной ячейки $7 \times 7$.

Рассмотрим количественные результаты, характеризующие уменьшение напряжений несоответствия при фазовом переходе. Данные о напряженном состоянии пленки установим для двух следующих границ раздела: 1) подложка кремния-псевдоморфная пленка Ge; 2) псевдоморфная пленка-поверхностная фаза германия. В центральной части наноостровка $\mathrm{Ge}(111)$ все четыре атомных слоя ПП подвергнуты биаксиальным однородным напряжениям сжатия $\sigma_{x x}=\sigma_{y y}$ (обозначим их $\left.\sigma_{1}\right)$. Оси $O x$ и $O y$ расположены параллельно границе раздела. Однако на каждый из трех атомных слоев ПФ кроме $\sigma_{1}$ действуют дополнительные нормальные напряжения. Эти дополнительные напряжения нижнего атомного слоя ПФ обозначим $\sigma_{2}$. Они равны перепаду напряжений между верхним атомным слоем ПП и нижним слоем ПФ. Напряжения $\sigma_{2}$ также примем однородными $\left(\sigma_{x x}=\sigma_{y y}\right)$. Для вычисления $\sigma_{1}$ воспользуемся формулой [3-5]:

$$
\sigma=2 G(1+v) f /(1-v) .
$$

Здесь $G$ - модуль сдвига, $v$ - коэффициент Пуассона. Важным членом формулы является параметр $f$ - 
относительное несоответствие решеток сопрягаемых кристаллов. В литературе нашли применение различные варианты записи параметра $f \quad[4,6,7]$. В общем виде зависимость $f$ от шести различных величин $f\left(a_{1}, a_{2}, G_{1}, G_{2}, v_{1}, v_{2}\right)$ представлена в [7]. Здесь $a-$ постоянная кристаллической решетки неискаженной (релаксированной) подложки $\left(a_{1}\right)$ и пленки $\left(a_{2}\right)$. $\mathrm{B}$ [7] обсуждаются частные случаи зависимости. Для наших экспериментальных исследований повышенный интерес представляют два варианта. В первую очередь это случай, когда упругие постоянные пленки и подложки являются практически одинаковыми: $\quad f_{1}=2\left(a_{1}-a_{2}\right) /\left(a_{1}+a_{2}\right)$. Согласно приложению, представленному в монографии [8], $v_{\mathrm{Ge}}=0.200, \quad G_{\mathrm{Ge}}=5.64 \cdot 10^{10} \mathrm{~N} / \mathrm{m}^{2}, \quad \nu_{\mathrm{Si}}=0.215$, $G_{\mathrm{Si}}=6.41 \cdot 10^{10} \mathrm{~N} / \mathrm{m}^{2}$, т.е. для германия и кремния несовпадение упругих постоянных менее $14 \%$. Во втором варианте основное внимание уделено геометрическому фактору. В наших исследованиях толщина подложки на несколько порядков величины превышает толщину пленки. Тогда в соответствии с результатом обсуждения рис. $1, a$ работы [7] $f_{2}=\left(a_{1}-a_{2}\right) / a_{2}$. Вычисленные величины параметров несоответствия составляют $f_{1}=-0.041$ и $f_{2}=-0.040$. Определенное по формуле (1) при среднем $f_{m}=-0.0405$ значение $\sigma_{1}$ равно $6.9 \cdot 10^{9} \mathrm{~N} / \mathrm{m}^{2}$.

Обсудим природу напряжений $\sigma_{2}$. Уже при формировании фазы $7 \times 7$ возникает релаксация напряжений несоответствия, поскольку атомная плотность ПФ $\rho_{7 \times 7}<\rho_{1 \times 1}$. При фазовом переходе $7 \times 7 \rightarrow 5 \times 5$ появляется дополнительная релаксация, так как $\rho_{5 \times 5}<\rho_{7 \times 7}$. Нижний слой ПФ (слой 3 на рис. 1,c) содержит систему вакансий, созданных массопереносом при образовании поверхностной фазы. Это приводит к уменьшению атомной плотности кристалла.

Для оценки релаксации напряжений несоответствия уменьшение плотности в некоторой степени уподобим эффекту растяжения атомного слоя. Поэтому при оценке уровня напряжений $\sigma_{2}$ воспользуемся понятием эффективного параметра несоответствия. К гетеросистеме $\mathrm{Ge}-\mathrm{Si}(111)$ аналогичное понятие было впервые применено в работе [9], где было рассчитано изменение упругой энергии гетеросистемы в рамках модели Ратша-Зангвила [10], согласно которой эффективный параметр несоответствия решеток уменьшается при переходе от нижнего слоя атомов к верхнему. Расчет $\sigma_{2}$ выполним сначала для фазы $7 \times 7$. Под параметром $f_{\text {eff }}^{7 \times 7}$ будем понимать относительную разницу средних межатомных расстояний в слое ПП и в нижнем атомном слое ПФ. В верхнем атомном слое ПП выберем площадку $S_{7 \times 7}$, которая по размеру совпадает с размером элементарной ячейки $7 \times 7$ и расположена строго под ней. Внутри площадки возьмем произвольный участок площадью $(48 / 49) S_{7 \times 7}$ и приложим к нему такое поле однородно растягивающих напряжений $\sigma_{2}$, чтобы площадь участка возросла до величины $S_{7 \times 7}$. Величина относительного увеличения площади равна
$\Delta S / S=1 / 49$, что приблизительно в 2 раза больше, чем $\Delta L / L$ - относительное линейное увеличение размера расширяющегося участка. Величина $\Delta L / L \approx 1 / 98$ (или $1.02 \%)$ и является эффективным параметром несоответствия $f_{e f f}^{7 \times 7}$. Подобным образом для ПФ $5 \times 5$ получим $f_{\text {eff }}^{5 \times 5} \approx 2.0 \%$. Эти несоответствия характеризуют однородные напряжения, которые являются растягивающими и, согласно формуле (1), равны $\sigma_{2}^{7 \times 7}=1.7 \cdot 10^{9} \mathrm{~N} / \mathrm{m}^{2}$ и $\sigma_{2}^{5 \times 5}=3.4 \cdot 10^{9} \mathrm{~N} / \mathrm{m}^{2}$. Тот факт, что $\sigma_{2}^{5 \times 5}>\sigma_{2}^{7 \times 7}$, означает, что в процессе фазового перехода $7 \times 7 \rightarrow 5 \times 5$ уровень нормальных напряжений в приповерхностном слое пленки уменьшается, что и свидетельствует об обсуждаемой в работе релаксации напряжений несоответствия. Отметим, что суммарные напряжения $\sigma_{1}+\sigma_{2}$ в нижнем (третьем) атомном слое ПФ остаются сжимающими, т.е. отрицательными. Для фаз $7 \times 7$ и $5 \times 5$ эти суммарные напряжения приблизительно равны $5.2 \cdot 10^{9}$ и $3.5 \cdot 10^{9} \mathrm{~N} / \mathrm{m}^{2}$ соответственно.

Для гетеросистемы $\mathrm{Ge}-\mathrm{Si}$, содержащей на поверхности пленки Ge фазу $7 \times 7$, величина результирующего несоответствия уменьшается от значения $f_{\mathrm{Ge}-\mathrm{Si}}$, равного $4 \%$, приблизительно до значения $3 \%$, что соответствует разности величин $f_{\mathrm{Ge}-\mathrm{Si}}-f_{\text {eff }}^{7 \times 7}$. Этот результат находится в согласии с экспериментальными данными, полученными с помощью комбинационного рассеяния [11]. Приведенная в работе формула (1) справедлива для сплошных псевдоморфных слоев пленки. В случае островков пленки зависимость существенно усложняется [12]. Мы использовали (1), поскольку средний латеральный размер островка, в котором наблюдается переход $7 \times 7 \rightarrow 5 \times 5$, в 30 раз и более превышает толщину пленки. При такой геометрии островка уровень нормальных напряжений $\sigma_{x x} \approx \sigma_{y y}$ в пленке максимален в ее центральной части и слабо отличается от величины, имеющей место в случае сплошной пленки. На периферии островка из-за присутствия боковых разгруженных участков боковой поверхности уровень $\sigma$ понижается. Тем не менее существенной релаксации на краях не происходит, поскольку края даже небольших островков $\mathrm{Ge}$ характеризуются напряженной структурой $7 \times 7$ [2] и не содержат элементы структуры $2 \times 8$, характерной для ненапряженного Ge.

В заключение отметим, что в настоящей работе на примере фазового перехода сверхструктуры $7 \times 7$ в $5 \times 5$ впервые показано, что наличие неплотных слоев поверхностной фазы способствует частичной релаксации напряжений несоответствия посредством поверхностного массопереноса избыточных атомов при формировании системы упорядоченных вакансий.

\section{Благодарности}

Авторы выражают благодарность А.В. Колесникову за помощь в подготовке статьи. 


\section{Финансирование работы}

Работа выполнена при поддержке Российского фонда фундаментальных исследований (грант № 16-29-03292).

\section{Конфликт интересов}

Авторы заявляют, что у них нет конфликта интересов.

\section{Список литературы}

[1] Kim H. // Atomic structure and defects of III-V compound semiconductor strained-layer-superlattices for infrared detection. Dissertation. Urbana, Illinois: University of Illinois at Urbana-Champaign, 2015. $125 \mathrm{p}$.

[2] Teys S.A. // Appl. Surf. Sci. 2017. V. 392. P. 1017-1025.

[3] Тхорик Ю.А., Хазан Л.С. // Пластическая деформация и дислокации несоответствия в гетероэпитаксиальных системах. Киев: Наук. думка, 1983. 135 с.

[4] Ayers J.E., Kujofsa T., Rago P., Raphael J.E. // Heteroepitaxy of semiconductors: theory, growth and characterization. 2nd ed. Boca Raton: Taylor \& Francis Group, 2017. P. 181.

[5] Скиба H.B. // Mater. Phys. Mech. 2014. V. 19. P. 68-87.

[6] Matthews J.W. // J. Vac. Sci. Technol. 1975. V. 12. P. 126-133.

[7] Romanov A.E., Wagner T. // Scripta Mater. 2001. V. 45. P. 325-331.

[8] Anderson P.M., Hirth J.P., Lothe J. // Theory of dislocations. N.Y.: University Press, 2017. $718 \mathrm{p}$.

[9] Кукушкин С.A., Осипов A.B., Schmitt F., Hess P. // ФТП. 2002. T. 36. B. 10. C. $1177-1185$.

[10] Ratsch C., Zangwill A. // Surf. Sci. 1993. V. 293. P. 123-131.

[11] Талочкин А.Б., Тийс С.А. // Письма в ЖЭТФ. 2002. Т. 75. B. 6. C. 314-317.

[12] Freund L.B., Suresh S. // Thin film materials, stress, defect formation and surface evolution. N.Y.: Univesity Press, 2003. $750 \mathrm{p}$. 